\title{
Emotional Reactivity and Parenting in Families Experiencing Homelessness
}

\author{
Alyssa R. Palmer ${ }^{1}$, Rachel A. Foster ${ }^{1}$, Rebecca Distefano ${ }^{2}$, and Ann S. Masten ${ }^{1}$ \\ ${ }^{1}$ University of Minnesota, Twin Cities \\ ${ }^{2}$ Teachers College, Columbia University
}

\begin{abstract}
Author Note
Alyssa R. Palmer: https://orcid.org/0000-0003-2365-4958

Rachel A. Foster: https://orcid.org/0000-0003-3307-1078

Rebecca Distefano: https://orcid.org/0000-0002-7536-4568

Ann S. Masten: http://orcid.org/0000-0003-2871-7508

Author for correspondence is Alyssa R Palmer, Institute of Child Development, University of Minnesota, 51 East River Parkway, Minneapolis, MN 55455; E-mail: palme719@umn.edu

We thank the children and families who participated in the research as well as Dr. Daniel Berry for his contribution to data collection and cleaning. This research was supported by a predoctoral fellowship from the National Institute of Health (T32 MH015755 to ARP), the University of Minnesota Interdisciplinary Fellowship (ARP), the Irving B. Harris Professorship in Child Development (ASM), and the Institute of Child Development Graduate Student Small Grants Program. The content is solely the responsibility of the authors and does not necessarily represent the official views of the National Institutes of Health. None of the authors have any conflicts of interest to declare.
\end{abstract}




\begin{abstract}
Parents are key protective systems in the lives of children experiencing homelessness. Evidence suggests that parent emotional reactivity, expression, and regulation play a critical role in promoting adaptive parenting behaviors. Studies of emotional reactivity in parents utilize different methods, including self-report, observations, and physiological measures. However, these methods are rarely evaluated together, particularly among disadvantaged families. The present study examined how subjective (i.e., self-report), observed, and physiological measures of parent emotional reactivity relate to each other and to observed parenting behaviors in parentchild interaction tasks comprised of problem-solving and teaching tasks. Participants included fifty 4- to 7-year-old children and their caregivers staying in an emergency homeless shelter. Observed and subjective emotional reactivity were largely uncorrelated, except in the case of positive emotions during problem-solving tasks. Adaptive parenting behavior was related to lower scores on measures of subjective and observed negative emotions and higher observed scores for positive emotions during problem-solving tasks, as well as higher observed scores of positive emotions during teaching tasks. Physiological reactivity was not related to parenting behaviors. Results suggest that associations of emotional reactivity with parenting behavior depend on the context of the parent-child interaction and how emotional reactivity is measured.
\end{abstract}

Keywords: Parenting; Emotion; RSA; Homelessness 


\section{Emotional Reactivity and Parenting in Families Experiencing Homelessness}

Families experiencing homelessness often encounter multiple adversities, including food insecurity, residential instability, community violence, and stressful shelter conditions (Haskett \& Armstrong, 2019). Children experiencing homelessness are more likely to have problems in academic achievement, mental health, and physical health (Bassuk et al., 2015; Masten et al., 2015). However, adaptive parenting behaviors consistently predict child adjustment in the context of homelessness (Cutuli \& Herbers, 2014). In social information learning theory (e.g., Forgatch \& Degarmo, 2005), the core components of adaptive parenting behaviors include skill encouragement, positive involvement, and problem-solving skills, as well as less coercive discipline. Skill encouragement is an ability to promote children's skills through contingent encouragement and scaffolding, and positive involvement includes behaviors like warmth, empathy, and affection. Problem-solving refers to generating feasible solutions to interpersonal conflicts, while coercive discipline highlights parental harsh/aversive behaviors. Among families experiencing homelessness, adaptive parenting behaviors are linked to fewer externalizing and internalizing symptoms in children (Labella et al., 2019; Palmer et al., 2020), as well as more peer acceptance and better cognitive functioning (Labella et al., 2019; Narayan et al., 2014).

Unfortunately, the extensive adversity associated with homelessness is likely to influence the functioning of parents as well as children (David et al., 2012; Perlman et al., 2012). According to family stress theory, economic pressure places parents at increased risk for emotional distress, which may undermine adaptive parenting behaviors (Conger \& Donnellan, 2007). This stress may be particularly salient when families have recently sought out emergency shelter. To foster positive development in children experiencing homelessness, it is important to understand factors underlying adaptive parenting in this acutely stressful context. 
One factor that may be related to parenting in the context of homelessness is parent emotional reactivity. Despite being in a highly strenuous housing situation, parents must manage their emotional expressions and maintain effective child rearing practices (Gewirtz et al., 2009). Labella et al. (2016) demonstrated that parents' expressed negativity (e.g., hostility) during an interview about their child was inversely related to child prosocial behavior. Despite endorsing high levels of internal distress, parents still demonstrated adaptive parenting behaviors. These results suggest that within the context of homelessness, children may benefit from parents' ability to modulate the outward expression of their emotional reactions and provide sensitive parenting. This also suggests that there may be differences in physiological and internal experience of emotions compared to external emotional expression.

Adaptive parenting is not only related to lower expressed negative emotions (Niehaus et al., 2019; Turpyn \& Chaplin, 2016), but also the maintenance and enhancement of positive emotions (Hajal et al., 2019; Julian et al., 2018). Unfortunately, negative and positive emotions rarely are studied in tandem, nor always with parenting context in mind. The role of positive emotional reactivity may be very different in a challenging parent-child interaction versus a playbased teaching interaction (Stifter \& Augustine, 2019). Interestingly, one study found that incongruent positive affect (i.e., positive emotions during a negatively-valenced interaction) was related to less sensitive parenting (Borelli et al., 2012). In the current study we evaluate the role of positive and negative emotions across multiple parenting contexts.

Parents' subjective emotional experiences may also differ from their physiological reactivity. The polyvagal theory (Porges, 2007) posits that parasympathetic nervous system functioning underlies emotional reactivity and regulation. Respiratory sinus arrhythmia (RSA) is used as a measure of parasympathetic nervous system activity. Higher resting RSA is related to 
adaptive emotional responses to environmental stimuli (Balzarotti et al., 2017) and adaptive parenting behaviors (e.g. Zhang et al., 2017). Larger RSA withdrawal (i.e., RSA decreases from baseline to task) during challenge tasks has been consistently related to better emotion regulation and fewer displays of negative emotions (Balzarotti et al., 2017). For positively-valenced social tasks, some evidence suggests that RSA augmentation (i.e., RSA increases from baseline to task) may be more indicative of adaptive social behaviors (Kok \& Fredrickson, 2010). Evaluating what unique information is gained from different measurements of emotional reactivity across contexts, is important to understanding the role of emotions in parenting.

We investigated the relations among three indicators of emotional reactivity across a baseline task and two types of parent-child interaction tasks typically used to rate adaptive parenting behaviors (e.g. Forgatch \& Degarmo, 1999). This included two problem-solving tasks where dyads addressed a family conflict and three game-like and play-based teaching tasks. We first evaluated whether the different tasks yielded different levels of subjective and observed positive and negative emotion as well as RSA reactivity. We hypothesized that problem-solving tasks would be more negatively valenced than teaching tasks (due to the play-based nature of the teaching tasks). Both tasks were expected to elicit more positive and negative emotional reactivity than the baseline task. Additionally, we hypothesized that, parents would engage in RSA withdrawal to the problem-solving tasks (representing a need for physiological regulation) and RSA augmentation during the teaching task (representing positive social engagement).

Our second aim was to evaluate whether positive and negative emotion scores across problem-solving and teaching tasks would differentially predict observed parenting behaviors. We hypothesized that lower observed and subjective negative emotions scores across all tasks would be related to more adaptive parenting behaviors. We also predicted that lower observed 
and subjective negative emotion scores as well as RSA withdrawal during the problem-solving tasks would be more strongly associated with a higher score on adaptive parenting behaviors compared to negative emotion scores and RSA augmentation during the teaching tasks. We expected that problem-solving tasks would be more likely to elicit negative emotions compared to teaching tasks and larger displays of negative emotions would be more likely to interfere with the goal of collectively solving a problem. Positive emotion scores were expected to have a larger positive effect on parenting behaviors during teaching tasks than during problem-solving tasks. Positive emotion was expected to be more common in the teaching task context and congruent with the goals of the teaching task compared to incongruent displays of positive affect in the problem-solving tasks. A third exploratory aim was to test whether the three distinct indicators of emotional reactivity (subjective, observed, and physiological) related to each other and uniquely predicted parenting behaviors in the context of homelessness.

\section{Method}

Families with a 4- to 7-year-old child were recruited from an urban emergency homeless shelter in a Midwestern U.S. city in 2017. Family eligibility criteria included (1) at least a three day stay in the shelter, (2) a sufficient understanding of English, and (3) no known developmental delay that would impede participation. This pilot study was a part of a larger study of families with data collected across two study sessions approximately one week apart that included 50 families who completed both visits. Of the 114 eligible families residing in the shelter during the recruitment period, 58 families participated in the study. The other 56 eligible families never learned of the study, were unavailable, or were not interested. All study procedures were approved by the (BLINDED) Institutional Review Board. Parents completed consent and children gave verbal assent. Study sessions took place in private research rooms 
located inside the emergency shelter. Trained examiners administered the parent measures in a separate room and read aloud all questions and instructions to alleviate concerns about literacy.

During the first session, parents completed a family information questionnaire, which included questions about family demographics and background. Caregivers were primarily biological mothers (84\%), identified as Black $(60 \%)$, and were $21-62$ years old $(M=32.50 ; S D$ =9.29). 15 parents did not finish high school, 16 had a high school diploma or equivalent, and 19 had post-secondary education or training. Children were 5.4 years old on average $(S D=0.7)$, and $42 \%$ female. All other measures were administered during the families' second visit.

During the second visit, parents wore a heart rate monitor (FirstBeat BodyGuard2) to capture RSA across two baseline tasks and parent-child interaction tasks. To capture resting RSA, parents spent five minutes quietly watching a video of nature photos. While in separate rooms, both the parent and child identified a challenging family conflict. Dyads then reunited and completed the interaction tasks. First, they participated in a five-minute active talking baseline task where the parent and child took turns describing pictures in a storybook. Parents were asked to turn the pages and point to pictures in the book to account for the effects of seated movement on RSA. Next, the dyad completed a 25-minute set of standardized Family Interaction Tasks (FITs) including two problem-solving discussions based on the distressing conflict they chose (attempting to solve one family problem picked by the caregiver and one picked by the child) and three teaching-oriented games (a guessing game, a marble labyrinth game, and tangram puzzles; see DeGarmo et al., 2004 and Gewirtz et al., 2009).

Parents rated their own emotions (happy, excited, frustrated, angry, and sad) after the talking baseline, the two problem-solving discussions and the three teaching games. The examiner presented the parent with a card representing the target emotion and a 5-point scale 
from 1 (not at all) to (very). Prior to the interaction tasks, the parent examiner had shown the cards to the parent and instructed them to only point to the number so that the child would not know how the parent was feeling during the dyadic tasks. Across the assessment points, ratings for happy and excited were averaged for one subjective positive emotion score ( $\alpha=0.76$ to 0.82 ) and ratings for frustrated, angry, and sad were averaged for one subjective negative emotion score $(\alpha=0.64$ to 0.82$)$.

Inter-beat interval (IBI) data were sampled at $1000 \mathrm{~Hz}$ with the FirstBeat BodyGuard 2 device. Research assistants who are reliable with Porges' gold standard (Brain Body Center, University of Illinois at Chicago) used CardioEdit to hand-edited aberrant IBI values. Cleaners adjusted IBI values that indicated a missed R-peak, a miss-labeled R-peak, or movement artifacts. Those values were replaced by an imputed IBI value based on IBI values surrounding the mis-identified beat. No more than fifteen percent of any file was corrected, with the majority of files requiring no editing. Fifteen percent of the IBI files were double-edited for reliability. RSA values were estimated using $0.14-0.40 \mathrm{~Hz}$ frequency band, representative of normative adult respiration using CardioBatch software (Brain Body Center, University of Illinois at Chicago). RSA means per parent per task were averaged across the two problem-solving discussions $(r=0.95, p<.001)$ and three teaching tasks, respectively $(r=0.92-0.97, p<.001)$. Physiological reactivity was calculated as the difference between the talking baseline average RSA and the average RSA from each set of interaction tasks. A positive value indicates RSA augmentation and a negative value indicates RSA withdrawal to the task. The larger the negative number, the more withdrawal or physiological regulation that is occurring during that task.

Two coders rated the parents' observed emotions and parenting behaviors. Twenty percent of videos were coded by both coders to determine reliability (ICC: .87-.94). Parents' 
emotions were rated on a 6-point scale from 0 (never) to 5 (always) on displays of negative and positive emotions after each problem-solving task and at the end of the teaching tasks. Three indicators (e.g., "seemed sad, blue, depressed”) were averaged for one observed negative emotion score ( $\alpha=0.60$ to 0.66$)$ and the single indicator of positive emotion ("seemed cheerful/pleasant") was used as the observed positive emotion score for each set of tasks.

Our observed measure of adaptive parenting behaviors was based on the Parent Management Training Oregon FITs coding scheme (DeGarmo et al., 2004) and adjusted for families experiencing homelessness to be more contextually and culturally sensitive (Forgatch et al., 2010). Parents were rated on a series of behaviors that indicated their positive involvement, problem-solving, inept coercive discipline (reverse-coded), and skill encouragement across all of the interaction tasks. These subscales were standardized and composited for one observed adaptive parenting score (Palmer, 2018).

Missingness ranged from $12 \%$ (subjective emotion ratings) to $26 \%$ (RSA during problem-solving tasks). Under the assumption that data are missing at random (MAR; Little et al., 2016), we used multiple imputation with the R program MICE to generate unbiased parameter estimates across 20 data sets using fully conditional specifications (R Core Team, 2020; van Buuren \& Groothuis-Oudshoorn, 2011). Missing RSA reactivity scores were addressed using passive imputation, which retains the relation between baseline RSA and interaction task RSA in the imputation model. The 20 imputed datasets were pooled using Rubin's rules which incorporate both within and between imputation variability (Rubin, 2004; Schafer \& Graham, 2002). In the subsequent analyses we used the imputed data, although the pattern of the results was similar using listwise deletion.

\section{Results}


First, we conducted a series of $t$-tests between the baseline, problem-solving, and teaching tasks to compare levels of emotional reactivity (Table 1). Compared to the baseline task, parents reported higher subjective negative emotion scores during problem-solving tasks and higher subjective positive emotion scores during teaching tasks. Parents also had higher scores on observed positive emotion during teaching tasks compared to problem-solving tasks.

Descriptive analyses suggest that observed and subjective emotional reactivity scores were largely uncorrelated with each other within task, except for measures of positive emotions during the problem-solving tasks ( $r=.46, p<.05$; see Supplemental Table 1 for correlations). It is important to note that there was less variability in negative emotion reactivity scores compared to positively emotional reactivity scores across tasks (Supplemental Table 2).

To further address the second and third aims, multiple linear regression was used to evaluate how different indicators of emotional reactivity during the problem-solving tasks and the teaching tasks related to overall adaptive parenting (Table 2). In line with hypotheses, lower subjective and observed negative emotion scores during the problem-solving tasks were related to a higher adaptive parenting score. Higher observed positive emotion scores during the problem-solving task were related to higher adaptive parenting score. Only higher observed positive emotions during the teaching tasks were positively related to adaptive parenting. Overall, results indicate that observed emotions were associated with observed ratings of adaptive parenting, even after controlling for subjective emotions. Physiological reactivity was unrelated to adaptive parenting scores.

\section{Discussion}

The current study adds to research on the antecedents of adaptive parenting in the context of homelessness. Results generally support the hypothesis that emotional reactivity is associated 
with adaptive parenting behaviors among parents experiencing homelessness. Given the pivotal role that parenting plays in supporting child adaptation in this context (e.g. Cutuli \& Herbers, 2014), these findings suggest that increasing parents' expressed positive emotions and decreasing negative emotional reactivity may bolster a critical resilience factor for young children.

The first aim of this study was to identify differences in emotional reactivity across tasks. Parent interaction tasks elicited more negative and positive emotions compared to a structured baseline interaction. Further, the teaching tasks tended to have higher positive emotion scores than the problem-solving tasks, suggesting that the teaching context may elicit more positive emotions. Future studies may benefit from including more exclusively positive tasks such as planning a fun activity or free play. Additionally, the problem-solving task had higher ratings of subjective negative emotions compared to baseline, but there were no differences in negative emotion between the problem-solving and teaching tasks. However, it is important to note that subjective and observed negative emotions were not frequently endorsed across tasks. It is possible that these tasks were not as negatively eliciting given the children's developmental stage and ability to engaged in a problem-solving discussion. Finally, there were no differences in physiological reactivity across the tasks.

The second aim of this study was to evaluate how various indicators of emotional reactivity across different parent-child interactions independently predicted parenting behaviors. Observed but not subjective positive emotions across tasks predicted higher adaptive parenting scores. There was a larger effect of positive emotion during the teaching tasks compared to positive emotion during the problem-solving tasks. Expressed positive emotions may facilitate the engagement of more adaptive parenting behaviors in positively valenced parenting contexts as well as more negatively valenced problem-solving discussions at this age. Positive emotional 
experiences can facilitate sensitive and responsive parenting behaviors in the moment and build a stronger relationship between parents and children via increased parent interest, attention, and intrinsic motivation to interact. Alternatively, the associations in this study could be conflated by shared method variance because observed emotions and parenting behaviors were rated by the same coders. Positive affect may influence how coders rate parenting behaviors, even if they are not explicitly a part of the parenting rating scale. As a sensitivity test, we evaluated the correlation from one coder's emotion rating to the other's parenting ratings on reliability videos and found similar patterns of association across and within raters (see Supplemental Table 3). Within this small interrater reliability subsample, the association of positive emotion with adaptive parenting appeared to not solely be attributable to bias or halo effects. Future studies should critically evaluate the existence of such biases in observational rating systems.

Higher subjective and observed negative emotional reactivity scores during the problemsolving tasks were related to lower adaptive parenting scores. However, negative emotional reactivity scores during the teaching tasks were not related to adaptive parenting. These results underscore the importance of considering which emotions are most salient for any given interaction context. Future studies may benefit from studying these phenomena in more emotionally-arousing contexts such as clean-up tasks or disciplinary interactions in comparison to free play.

Although observed and subjective emotion scores showed similar relational patterns to parenting behaviors, they had unique predictive variances, suggesting that they provide different information about emotional processes in parenting. Subjective and observed emotions may be separable constructs, representing differences in how a parent feels and their expression of those feelings. However, contrary to expectations, once the effects of observed and subjective 
emotions were controlled, physiological reactivity (indexed by RSA, an internal physiological indicator of emotional reactivity) was not related to parenting behaviors. Effects of RSA on parenting behaviors could be small and may be mediated by the ultimate interpretation of physiological responses based on a parent's subjective emotional experience. Further, research on dynamic patterns of physiological responding across task and parent-child RSA synchrony suggests that ideal patterns of regulation may fluctuate moment-to-moment across a task. Parentchild physiological synchrony may be a more salient biological predictor of adaptive parenting behaviors than individual parent reactivity (e.g., Giuliano et al., 2015; Lunkenheimer et al., 2017). Future work could analyze second-by-second RSA and emotion expression to inform how dynamic emotional reactivity and physiological reactivity are related across parenting tasks.

A key strength of this study was including multiple indicators of emotional reactivity within a set of standardized parenting tasks. It was particularly novel to include physiological measures with families experiencing homelessness. Given that this pilot study yielded promising results, future studies with more participants are warranted. This study was only powered to find medium to large effects, and not powered to test for mediation. For example, it will be particularly important to understand moderators, such as current levels of life stress and distress, as well as the impact of system-level financial supports and psycho-social interventions on parent emotional wellbeing. Additional research is needed to further examine the role of positive and negative emotions in risk and resilience processes for parenting in the context of homelessness and identify ways that emotional reactivity might be leveraged as an intervention target to promote parenting behaviors and thereby child wellbeing. 


\section{References}

Balzarotti, S., Biassoni, F., Colombo, B., Ciceri, M. R. (2017). Cardiac vagal control as a marker of emotion regulation in healthy adults: A review. Biological Psychology, 130, 5466. https://doi.org/10.1016/j.biopsycho.2017.10.008

Bassuk, E. L., Richard, M. K., \& Tsertsvadze, A. (2015). The prevalence of mental illness in homeless children: A systematic review and meta-analysis. Journal of the American Academy of Child \& Adolescent Psychiatry, 54, 86-96.

https://doi.org/10.1016/j.jaac.2014.11.008

Borelli, J. L., West, J. L., Decoste, C., \& Suchman, N. E. (2012). Emotionally avoidant language in the parenting interviews of substance-dependent mothers: Associations with reflective functioning, recent substance use, and parenting behavior. Infant Mental Health Journal, 33(5), 506-519. doi:10.1002/imhj.21340

Brain-Body Center, University of Illinois at Chicago. 2007.

Conger, R. D., \& Donnellan, M. B. (2007). An interactionist perspective on the socioeconomic context of human development. Annual Review of Psychology, 58, 175-199. doi:10.1146/annurev.psych.58.110405.085551

Cutuli, J. J., \& Herbers, J. E. (2014). Promoting resilience for children who experience family homelessness: Opportunities to encourage developmental competence. Cityscape, 16, 113-140. https://doi.org/10.1007/978-1-4614-8718-0_10

David, D. H., Gelberg, L., \& Suchman, N. E. (2012). Implications of homelessness for parenting young children: A preliminary review from a developmental attachment perspective. Infant Mental Health Journal, 33, 1-9. https://doi.org/10.1002/imhj.20333 
DeGarmo, D. S., Patterson, G. R., \& Forgatch, M. S. (2004). How do outcomes in a specified parent training intervention maintain or wane over time? Prevention Science, 5(2), 73-89. doi:10.1023/b:prev.0000023078.30191.e0

Forgatch, M. S., Plowman, E. J., Gewirtz, A. H., \& Stubbs, J. (2010). Observed family interaction code training manual (Unpublished manual.). Minneapolis, MN: University of Minnesota.

Gewirtz, A. H., DeGarmo, D. S., Plowman, E. J., August, G., \& Realmuto, G. (2009). Parenting, parental mental health, and child functioning in families residing in supportive housing. American Journal of Orthopsychiatry, 79(3), 336-347. http://dx.doi.org.ezp3.lib.umn.edu/10.1037/a0016732

Giuliano, R. J., Skowron, E. A., \& Berkman, E. T. (2015). Growth models of dyadic synchrony and mother-child vagal tone in the context of parenting at-risk. Biological Psychology, 105, 29-36. https://doi.org/10.1016/j.biopsycho.2014.12.009

Hajal, N. J., Teti, D. M., Cole, P. M., \& Ram, N. (2019). Maternal emotion, motivation, and regulation during real-world parenting challenges. Journal of Family Psychology, 33(1), 109-120. doi:10.1037/fam0000475

Haskett, M. E., \& Armstrong, J. M. (2019). The experience of family homelessness. In B.H. Jouriles \& M.A. Whisman (Eds.), APA Handbook of Contemporary Family Psychology: Applications and broad impact of family psychology (pp. 523-538). Washington, DC: American Psychological Association. doi:10.1037/0000100-032

Herbers, J., Garcia, E., \& Obradović, J. (2017). Parenting assessed by observation versus parentreport: Moderation by parent distress and family socioeconomic status. Journal of Child and Family Studies, 26(12), 3339-3350. https://doi.org/10.1007/s10826-017-0848-8 
Julian, M. M., Muzik, M., Kees, M., Valenstein, M., Dexter, C., \& Rosenblum, K. L. (2018). Intervention effects on reflectivity explain change in positive parenting in military families with young children. Journal of Family Psychology, 32(6), 804-815. doi:10.1037/fam0000431

Kok, B. E., \& Fredrickson, B. L. (2010). Upward spirals of the heart: Autonomic flexibility, as indexed by vagal tone, reciprocally and prospectively predicts positive emotions and social connectedness. Biological psychology, 85(3), 432-436.

https://doi.org/10.1016/j.biopsycho.2010.09.005

Labella, M. H., Narayan, A. J., \& Masten, A. S. (2016). Emotional climate in families experiencing homelessness: Associations with child affect and socioemotional adjustment in school. Social Development, 25(2), 304-321. doi:10.1111/sode.12154

Labella, M. H., Narayan, A. J., McCormick, C. M., Desjardins, C. D., \& Masten, A. S. (2019). Risk and adversity, parenting quality, and children's social-emotional adjustment in families experiencing homelessness. Child Development, 90(1), 227-244. https://doi.org/10.1111/cdev.12894

Leerkes, E. M., \& Augustine, M. E. (2019). Parenting and emotions. In M. Bornstein (Eds.), Handbook of Parenting, (pp. 620-653). New York, NY, Routledge. doi:10.4324/9780429433214-18

Little, T. D., Lang, K. M., Wu, W., \& Rhemtulla, M. (2016). Missing data. Developmental Psychopathology, 1-37. https://doi.org/10.1002/9781119125556.devpsy117

Lunkenheimer, E., Tiberio, S. S., Skoranski, A. M., Buss, K. A., \& Cole, P. M. (2018). Parentchild coregulation of parasympathetic processes varies by social context and risk for psychopathology. Psychophysiology, 55(2), e12985. https://doi.org/10.1111/psyp.12985 
Masten, A. S., Fiat, A. E., Labella, M. H., \& Strack, R. A. (2015). Educating homeless and highly mobile students: Implications of research on risk and resilience. School Psychology Review, 44, 315-330. https://doi.org/10.17105/spr-15-0068.1

Masten, A.S., \& Palmer, A.R. (2019). Parenting to promote resilience in children. In M.H. Bornstein (Eds.), Handbook of parenting: Vol 5: Parenting practices ( $\left.{ }^{\text {rd }} \mathrm{ed}, 156-188\right)$. Routledge Publisher. https://doi.org/10.4324/9780429401695-6

Narayan, A. J., Sapienza, J. K., Monn, A. R., Lingras, K. A., \& Masten, A. S. (2014). Risk, vulnerability, and protective processes of parental expressed emotion for children's peer relationships in contexts of parental violence. Journal of Clinical Child \& Adolescent Psychology, 44, 676-688. doi:10.1080/15374416.2014.881292

Niehaus, C. E., Chaplin, T. M., Turpyn, C. C., \& Gonçalves, S. F. (2019). Maternal emotional and physiological reactivity: implications for parenting and the parenting-adolescent relationship. Journal of Child and Family Studies, 28(3), 872-883. doi:10.1007/s10826018-01318-Z

Palmer, A.R. (2018). Coder Impression Technical Report Family Interaction Task SSIM17. Unpublished Technical Report

Palmer, A.R., Labella, M., Plowman, E.J., Foster, R. \& Masten, A.S. (2020). Parent emotion regulation strategies and parenting quality predict child internalizing symptoms in families experiencing homelessness. Social Development. 29, 732-749. https://doi.org/10.1111/sode.12435

Pereira, A. I., Barros, L., Roberto, M. S., \& Marques, T. (2017). Development of the Parent Emotion Regulation Scale (PERS): Factor structure and psychometric qualities. Journal of Child and Family Studies, 26(12), 3327-3338. doi:10.1007/s10826-017-0847-9 
Perlman, S., Cowan, B., Gewirtz, A., Haskett, M., \& Stokes, L. (2012). Promoting positive parenting in the context of homelessness. American Journal of Orthopsychiatry, 82(3), 402-412. doi:10.1111/j.1939-0025.2012.01158.x

Porges, S. W. (2007). The polyvagal perspective. Biological Psychology, 74, 116-143. doi:10.1016/j.biopsycho.2006.06.009

R Development Core Team (2020). R: A language and environment for statistical computing. Vienna, Austria: R Foundation for Statistical Computing.

Rubin, D. B. (2004). Multiple imputation for nonresponse in surveys (Vol. 81). New York: John Wiley \& Sons. https://doi.org/10.1002/9780470316696

Schafer, J. L., \& Graham, J. W. (2002). Missing data: Our view of the state of the art. Psychological Methods, 7, 147-177. doi:10.1037//1082-989x.7.2.147

Stifter C., Augustine M. (2019) Emotion regulation. In: LoBue V., Pérez-Edgar K., Buss K. (Eds) Handbook of Emotional Development (405-430). New York, NY. Springer, Cham. https://doi.org/10.1007/978-3-030-17332-6_16

Turpyn, C. C., \& Chaplin, T. M. (2015). Mindful parenting and parents' emotion expression: Effects on adolescent risk behaviors. Mindfulness, 7(1), 246-254. doi:10.1007/s12671$015-0440-5$

van Buuren S, Groothuis-Oudshoorn K (2011). “mice: Multivariate Imputation by Chained Equations in R.” Journal of Statistical Software, 45(3), 1 67. https://www.jstatsoft.org/v45/i03/.

Zhang, X., Cui, L., Han, Z. R., \& Yan, J. (2017). The heart of parenting: Parent HR dynamics and negative parenting while resolving conflict with child. Journal of Family Psychology, 31(2), 129. https://doi.org/10.1037/fam000028 


\section{Tables}

Table 1.

$t$-test of Differences Between Emotion Ratings Between Tasks

\begin{tabular}{cccc}
\hline & Problem-Solving & $\boldsymbol{t}(\boldsymbol{d} \boldsymbol{f})$ & $\boldsymbol{p}$ \\
\hline Talking Baseline & & .69 \\
& Subjective Positive Emotions & $0.40(29.95)$ & .02 \\
& Subjective Negative Emotions & $-2.46(40.84)$ & .57 \\
\hline Talking Baseline & Average RSA & $-0.57(37.16)$ & \\
\hline & Teaching Task & & .006 \\
& Subjective Positive Emotions & $-2.93(32.85)$ & .47 \\
& Subjective Negative Emotions & $-0.72(41.36)$ & .64 \\
\hline Problem-Solving & Average RSA & $-0.48(37.77)$ & .003 \\
& Teaching Task & & .12 \\
& Subjective Positive Emotions & $-3.12(38.88)$ & .01 \\
& Subjective Negative Emotions & $1.57(39.20)$ & .15 \\
& Observed Positive Emotions & $-2.72(32.86)$ & .95 \\
& Observed Negative Emotions & $1.49(35.03)$ & .76 \\
\hline
\end{tabular}


Table 2.

Parenting Behaviors Regressed on Emotional Reactivity During the Problem-Solving and Teaching Tasks

\begin{tabular}{|c|c|c|c|c|c|c|}
\hline 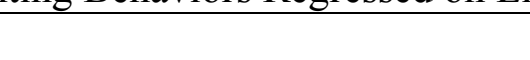 & \multicolumn{3}{|c|}{ Problem-Solving Tasks } & \multicolumn{3}{|c|}{ Teaching Tasks } \\
\hline & $B$ & $S E$ & $t$ & $B$ & $S E$ & $t$ \\
\hline Intercept & 2.67 & 0.20 & $12.78 * * *$ & 2.01 & 0.22 & $8.99 * * *$ \\
\hline High School Diploma & -0.01 & 0.11 & -0.08 & -0.12 & 0.11 & -1.10 \\
\hline Post-Secondary Education & 0.01 & 0.12 & 0.11 & -0.03 & 0.11 & -0.24 \\
\hline Subjective Positive Emotions & -0.06 & 0.03 & -1.63 & -0.07 & 0.04 & -1.68 \\
\hline Subjective Negative Emotions & -0.18 & 0.06 & $-2.60 *$ & 0.08 & 0.12 & 0.59 \\
\hline Observed Positive Emotions & 0.25 & 0.06 & $4.18 * * *$ & 0.38 & 0.05 & $7.77 * * *$ \\
\hline Observed Negative Emotions & -0.26 & 0.12 & $-2.09 *$ & 0.20 & 0.10 & $6.82^{\tau}$ \\
\hline Resting Baseline RSA & -0.04 & 0.03 & -1.44 & -0.04 & 0.02 & -1.55 \\
\hline RSA Reactivity & -0.04 & 0.07 & -0.52 & -0.12 & 0.09 & 1.30 \\
\hline & $2^{2}=0.6$ & 41,0 . & & $\mathrm{R}^{2}=0$. & 43,0 . & \\
\hline
\end{tabular}

Note: ${ }^{\tau} p<.10,{ }^{*} p<.05, * * p<.01, * * * p<.001$. 


\section{Supplementary Materials}

\section{Supplemental Table 1}

Key Variable Pearson Correlations

\begin{tabular}{|c|c|c|c|c|c|c|c|c|c|c|c|c|c|c|c|}
\hline & 1 & 2 & 3 & 4 & 5 & 6 & 7 & 8 & 9 & 10 & 11 & 12 & 13 & 14 & 15 \\
\hline \multicolumn{16}{|l|}{ 1. Age } \\
\hline 2. Sex & $0.50 * * *$ & & & & & & & & & & & & & & \\
\hline 3. Parenting & -0.06 & 0.01 & & & & & & & & & & & & & \\
\hline 4. RSA Resting Baseline & $-0.32 *$ & $-0.35^{*}$ & -0.02 & & & & & & & & & & & & \\
\hline 5. RSA PS Reactivity & 0.19 & -0.02 & -0.12 & -0.10 & & & & & & & & & & & \\
\hline 6. RSA TT Reactivity & 0.08 & 0.06 & -0.24 & -0.10 & $0.82 * * *$ & & & & & & & & & & \\
\hline 7. Subjective Positive TB & -0.01 & 0.07 & -0.04 & 0.18 & 0.12 & 0.19 & & & & & & & & & \\
\hline 8. Subjective Negative TB & 0.10 & 0.10 & -0.23 & -0.26 & 0.12 & 0.07 & $-0.32 *$ & & & & & & & & \\
\hline 9. Subjective Positive PS & -0.07 & -0.02 & 0.26 & 0.26 & 0.01 & 0.09 & $0.49 * *$ & -0.19 & & & & & & & \\
\hline 10. Subjective Negative PS & 0.20 & 0.08 & $-0.47 * *$ & $-0.33^{*}$ & 0.22 & 0.09 & -0.01 & $0.41 * *$ & $-0.46^{* *}$ & & & & & & \\
\hline 12. Subjective Negative TT & -0.14 & -0.17 & 0.27 & -0.20 & 0.13 & 0.26 & 0.17 & -0.02 & 0.25 & -0.12 & $-0.33^{*}$ & & & & \\
\hline 13. Observed Positive PS & -0.20 & -0.17 & $0.77 * * *$ & 0.14 & -0.12 & -0.20 & 0.09 & -0.26 & $0.46^{* *}$ & $-0.42 * *$ & 0.06 & $0.45^{* *}$ & & & \\
\hline 14. Observed Negative PS & -0.08 & -0.01 & $-0.49 * * *$ & -0.05 & -0.28 & -0.08 & -0.21 & -0.01 & -0.14 & -0.02 & -0.09 & $-0.32 *$ & $-0.40 * *$ & & \\
\hline 15. Observed Positive TT & 0.05 & 0.04 & $0.80 * * *$ & 0.14 & 0.01 & -0.09 & 0.10 & -0.22 & $0.32 *$ & $-0.33^{*}$ & 0.20 & 0.06 & $0.59 * * *$ & $-0.42 * *$ & \\
\hline 16. Observed Negative TT & -0.22 & -0.04 & -0.29 & -0.25 & -0.21 & 0.04 & -0.17 & -0.04 & -0.21 & 0.10 & -0.21 & 0.16 & -0.09 & $0.35^{*}$ & $-0.58 * * *$ \\
\hline
\end{tabular}

Note: PS: Problem Solving; TT: Teaching Task; TB: Talking Baseline; $* p<.05, * * p<.01,{ }^{* * *} p<.001$. 


\section{Supplemental Table 2}

\section{Descriptive Statistics}

\begin{tabular}{|c|c|c|c|}
\hline & Mean & $S D$ & Range \\
\hline Age & 32.50 & 9.29 & $21-62$ \\
\hline Male Parent & $n=6(12 \%)$ & & \\
\hline HS Diploma & $n=16(32 \%)$ & & \\
\hline Post-Secondary & $n=19(38 \%)$ & & \\
\hline Parenting Skills & 2.88 & 0.43 & $1.94-3.95$ \\
\hline Resting RSA & 0.00 & 1.59 & $2.82-3.06$ \\
\hline ive Positive TB & 2.31 & 0.97 & $0-4$ \\
\hline ve Negative TB & 0.24 & 0.59 & $0-3.67$ \\
\hline A Reactivity PS & 0.03 & 0.65 & $-1.30-0.91$ \\
\hline tive Positive PS & 2.23 & 1.35 & $0-4.0$ \\
\hline ve Negative PS & 0.52 & 0.76 & $0-3.67$ \\
\hline ved Positive PS & 2.15 & 0.99 & $0-4.5$ \\
\hline ed Negative PS & 0.48 & 0.44 & $0-1.83$ \\
\hline A Reactivity TT & -0.03 & 0.48 & $-1.77-1.87$ \\
\hline ive Positive TT & 2.84 & 1.07 & $0-4.0$ \\
\hline ve Negative TT & 0.29 & 0.50 & $0-2.67$ \\
\hline ved Positive TT & 2.74 & 1.03 & $0-5.00$ \\
\hline ed Negative TT & 0.38 & 0.46 & $0-2.33$ \\
\hline
\end{tabular}

Subjective Positive TB

Subjective Negative TB

RSA Reactivity PS

Subjective Positive PS

Subjective Negative PS

Observed Positive PS

Observed Negative PS

RSA Reactivity TT

Subjective Positive TT

Subjective Negative TT

Observed Positive TT

0.38

$1.94-3.95$

$82-3.06$$$
0-3.67
$$

$1.30-0.91$

$0-4.0$

$0-3.67$

$0-4.5$

$0-1.83$

Observed Negative TT

0.46

$0-2.33$

Note: HS is high school, PS is problem-solving, TT is teaching task, TB is talking baseline 


\section{Supplemental Table 3}

Pearson Correlations Between Observed Parenting Codes and Emotion Codes Between Coders $(n=10)$.

\begin{tabular}{|cc|c}
\hline & $\begin{array}{c}\text { Adaptive } \\
\text { Parenting } \\
\text { Coder 1 }\end{array}$ & $\begin{array}{c}\text { Adaptive } \\
\text { Parenting } \\
\text { Coder 2 }\end{array}$ \\
\hline Observed Negative Problem-Solving Coder 1 & -0.13 & -0.16 \\
Observed Positive Problem-Solving Coder 1 & $0.71 *$ & $0.65 *$ \\
Observed Negative Teaching Tasks Coder 1 & 0.16 & -0.05 \\
Observed Positive Teaching Tasks Coder 1 & $0.84 * *$ & $0.85^{* *}$ \\
\hline Observed Negative Problem-Solving Coder 2 & -0.49 & -0.58 \\
Observed Positive Problem-Solving Coder 2 & $0.82^{* *}$ & $0.92^{* *}$ \\
Observed Negative Teaching Tasks Coder 2 & -0.03 & -0.19 \\
Observed Negative Teaching Tasks Coder 2 & $0.64 *$ & $0.83^{* *}$ \\
\hline
\end{tabular}

Note: ${ }^{* *} p<.01, * p<.05$ 